\title{
Tuning Valley Degeneracy with Band Inversion
}

\author{
Michael Y. Toriyama, ${ }^{a *}$ Madison K. Brod,${ }^{a}$ Lídia C. Gomes, ${ }^{b}$ Ferdaushi A. Bipasha, ${ }^{b}$ Badih A. \\ Assaf, ${ }^{c}$ Elif Ertekin, ${ }^{b}$ and G. Jeffrey Snyder ${ }^{a *}$
}

Valley degeneracy is a key feature of the electronic structure that benefits the thermoelectric performance of a material. Despite recent studies which claim that high valley degeneracy can be achieved with inverted bands, our survey of rock-salt IV-VI compounds demonstrates that mere band inversion is an insufficient condition for high valley degeneracy; rather, there is a critical degree to which the bands must be inverted to induce multiple carrier pockets. The so-called "band inversion parameter" is a chemically-tunable parameter, offering a design route to achieving high valley degeneracy in compounds with inverted bands. We predict that the valley degeneracy of rock-salt IV-VI compounds can be increased from $N_{V}=4$ to $N_{V}=24$, which could result in a corresponding increase in the thermoelectric figure of merit $z T$.

\section{Introduction}

Engineering high valley degeneracy is a common design strategy for achieving high thermoelectric performance, as it enables high electrical conductivity while simultaneously maintaining high thermopower. ${ }^{1-6}$ In the absence of intervalley scattering, the thermoelectric quality factor, which determines the maximum thermoelectric figure of merit $z T$, is directly proportional to the valley degeneracy $N_{V} .{ }^{7}$ Improving $z T$ by increasing the valley degeneracy has been a focus of many studies; for example, converging the valence bands of PbTe at the L-point $\left(N_{V}=4\right)$ and along the $\Sigma$-line $\left(N_{V}=12\right)$ has been shown to improve the thermoelectric performance of $p$-type PbTe. ${ }^{8-13}$

Recently, nontrivial electronic structure topologies have also attracted interest as a strategy for increasing the valley degeneracy in materials such as rock-salt SnSe, ${ }^{14,15} \mathrm{PbTe}-\mathrm{SnTe}$-GeTe alloys, ${ }^{16}$ and $\mathrm{Bi}_{2} \mathrm{Te}_{3} \cdot{ }^{17-19}$ In these systems, it is often discussed that band inversion leads to highly-corrugated Fermi surfaces with multiple carrier pockets near the band edge. It is curious however that band inversion leads to high valley degeneracy in some materials while it does not in others; for example, $\mathrm{Bi}_{2} \mathrm{Te}_{3}$ has inverted bands and exhibits high valley degeneracy $\left(N_{V}=6\right),{ }^{20}$ whereas the chemically similar $\mathrm{Bi}_{2} \mathrm{Se}_{3}$ also has inverted bands but exhibits a single carrier pocket at the $\Gamma$-point $\left(N_{V}=1\right) .{ }^{18,19}$ While band inversion is a key characteristic of topological insulators in the context of surface/edge states, ${ }^{21-25}$ comparatively little is discussed regarding the consequent electronic structure in the bulk.

Here, we discuss the roles of band inversion on the valley degeneracy of rock-salt IV-VI compounds using a combination of density functional theory (DFT) calculations, $k \bullet p$ theory, and the tight binding method. We show that mere band inversion is an

${ }^{a}$ Department of Materials Science and Engineering, Northwestern University, Evanston, IL 60208.

${ }^{b}$ Department of Mechanical Science and Engineering, University of Illinois at UrbanaChampaign, Urbana, IL 61801.

${ }^{c}$ Department of Physics, University of Notre Dame, Notre Dame, IN 46556.

*E-mail: MichaelToriyama2024@u.northwestern.edu, jeff.snyder@northwestern.edu insufficient condition to obtain a valley degeneracy higher than $N_{V}=4$ in rock-salt IV-VI compounds, and that the degree to which the bands are inverted instead determines whether $N_{V}>4$ can be achieved. To this end, we show that band inversion can not only be induced, but also modified chemically by tuning the nearestneighbor sp-interaction in rock-salt IV-VI compounds. We suggest possible avenues of controlling band inversion in rock-salt IV-VI compounds, leading to an overall $6 \times$ increase in the valley degeneracy from $N_{V}=4$ to $N_{V}=24$.

\section{Electronic Structure Topologies of Rock- Salt IV-VI Compounds}

Binary IV-VI compounds in the rock-salt crystal structure can exhibit either bands with normal ordering or inverted bands. The band structures and Fermi surfaces of IV-VI compounds are shown in Figures 1 and S1. The partial contributions of atomic orbitals to each band suggest that SnTe, SnSe, SnS, GeSe, and GeS in the rock-salt phase have inverted bands at the L-point of the Brillouin zone, while $\mathrm{PbTe}, \mathrm{PbSe}, \mathrm{PbS}$, and $\mathrm{GeTe}$ do not, in agreement with previous studies. ${ }^{26,27}$

All compounds with normal band ordering exhibit a single carrier pocket at the L-point for both the conduction and valence bands, whereas the shapes of the band edges are more diverse for compounds with inverted bands. For example, while SnTe exhibits a single carrier pocket for the conduction and valence bands centered at each L-point (Figure 1a), each half-pocket breaks into three full carrier pockets that are offset from the $L$-plane (i.e. the hexagonal zone boundary of the Brillouin zone containing the L-point) in rock-salt SnSe and SnS (Figures 1b, S1f), resulting in an overall $6 \times$ increase in the valley degeneracy from $N_{V}=4$ to $N_{V}=24$. Additionally, rock-salt GeSe and GeS both exhibit a toroidal conduction band Fermi surface (Figures 1c and S1e) as opposed to a single conduction band pocket.

It is interesting that similar compounds with inverted bands exhibit diverse Fermi surface geometries, some with a single pocket centered at each L-point and others with multiple carrier pockets 
(a)
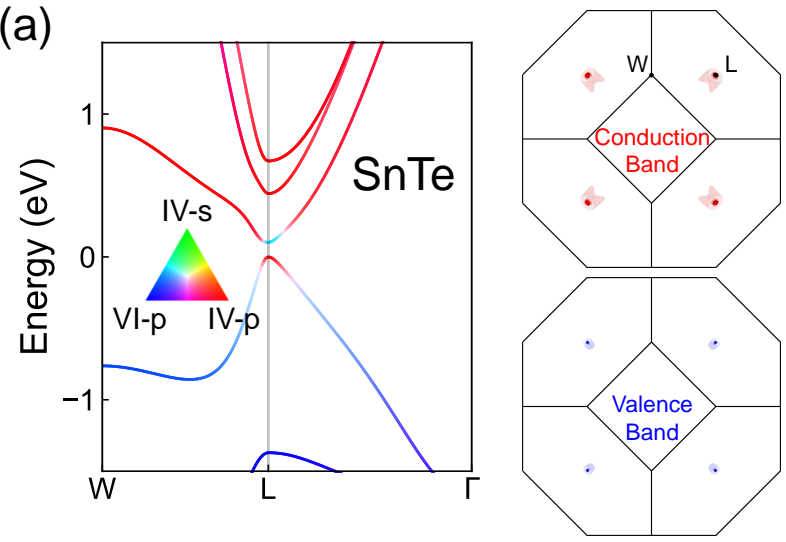

(b)
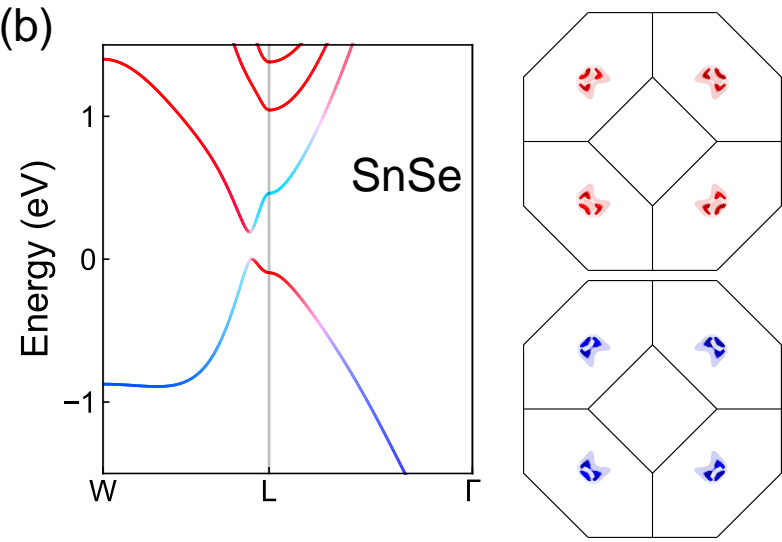

(c)

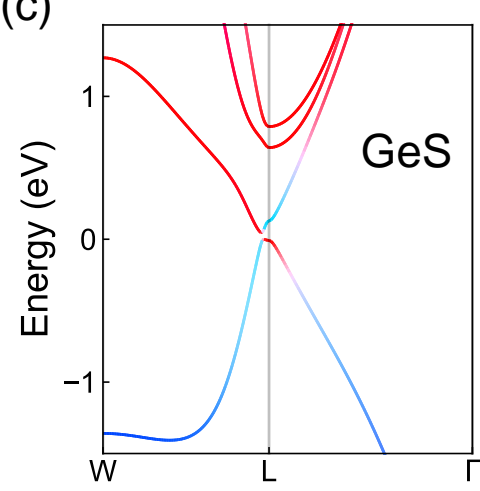

\section{When Does Band Inversion Lead to High Valley Degeneracy?}

$k \bullet p$ perturbation theory uses the lowest order dispersion relation consistent with the symmetry of the structure to model the electronic bands at or near a single $k$-point, ${ }^{28}$ such as the L-point in IV-VI semiconductors. ${ }^{29-32}$ The $k \bullet p$ model for the L-point with $\overline{3} m$-symmetry is given in the Methods section, where the parameters are categorized into those related to interactions parallel to the L-plane (i.e. in-plane interactions, denoted by $\|$ ) and interactions perpendicular to the L-plane (i.e. out-of-plane interactions, denoted by $\perp$ ). The degree to which the bands are inverted is parametrized by $M_{0}$, which we refer to as the "band inversion parameter" since $M_{0}>0$ yields normal bands (Figure 2a), $M_{0}=0$ yields Dirac cones (Figure $2 \mathrm{~b}$ ), and $M_{0}<0$ gives inverted bands (Figures $2 \mathrm{c}$ and $2 \mathrm{~d}$ ). While both in-plane and out-of-plane interactions are necessary to describe the carrier pockets in e.g. rocksalt SnSe, we begin our discussion with in-plane interactions to understand the role of the band inversion parameter $M_{0}$ for valley degeneracy.

To obtain high valley degeneracy, the band extrema must be offset from the high-symmetry L-point, meaning the curvature of the band should be reversed at the L-point in the direction of the true band extrema. As derived in the Methods section, the curvatures of the in-plane energy bands for $M_{0}<0$ are

$$
\begin{aligned}
& \left.\frac{\partial^{2} E_{V}^{\mathrm{L}-\text { plane }}}{\partial \mathbf{k}^{2}}\right|_{\mathbf{k}=\mathrm{L}}=2 C_{\|}+\left(2 M_{\|}-\frac{A_{\|}^{2}}{\left|M_{0}\right|}\right) \\
& \left.\frac{\partial^{2} E_{C}^{\mathrm{L}-\text { plane }}}{\partial \mathbf{k}^{2}}\right|_{\mathbf{k}=\mathrm{L}}=2 C_{\|}-\left(2 M_{\|}-\frac{A_{\|}^{2}}{\left|M_{0}\right|}\right)
\end{aligned}
$$

where $E_{V}^{\mathrm{L}-\text { plane }}$ and $E_{C}^{\mathrm{L}-\text { plane }}$ denote the valence and conduction bands, respectively, along a path on the L-plane containing the L-point (i.e. $k_{3}=0$ ). As a result, high valley degeneracy in both bands, in which $\left.\frac{\partial^{2} E_{V}^{\mathrm{L}-\text { plane }}}{\partial \mathbf{k}^{2}}\right|_{\mathbf{k}=\mathrm{L}}>0$ and $\left.\frac{\partial^{2} E_{C}^{\mathrm{L}-\text { plane }}}{\partial \mathbf{k}^{2}}\right|_{\mathbf{k}=\mathrm{L}}<0$, can be achieved provided that

$$
2 M_{\|}>\frac{A_{\|}^{2}}{\left|M_{0}\right|}+2\left|C_{\|}\right| .
$$

Therefore, if the bands are inverted but Equation (2) is not satisfied, then the conduction and valence bands are single-valleyed at the point of inversion (Figure 2c). On the other hand, if the bands are inverted and Equation (2) is satisfied, then the bands form ring-like edges around the point of inversion (Figure 2d). The threshold can be satisfied by increasing the degree to which the bands are inverted (i.e. by making $M_{0}$ more negative).

The six $\overline{3} m$-symmetric carrier pockets which are offset from the L-plane in rock-salt SnSe and SnS (Figures 1b, S1f) indicate that out-of-plane interactions are necessary to properly model the electronic structures of these compounds. As shown in Figure 3, the $k \bullet p$ model can capture the general electronic structure features of all rock-salt IV-VI compounds when in-plane and out-ofplane interactions are included. A single carrier pocket is possible regardless of whether the bands are normal (Figure 3a) or in- 
(a)

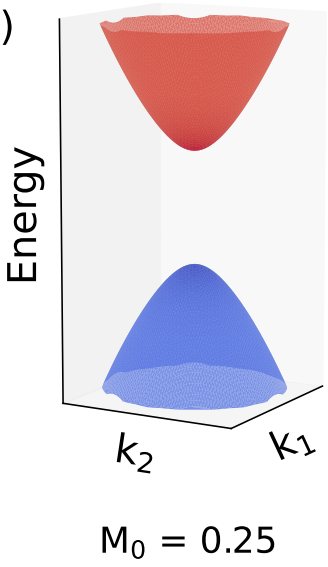

(b)

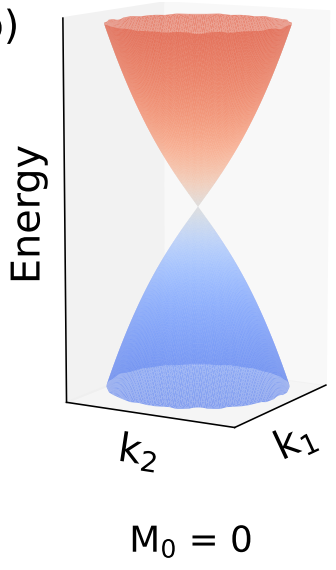

(c)

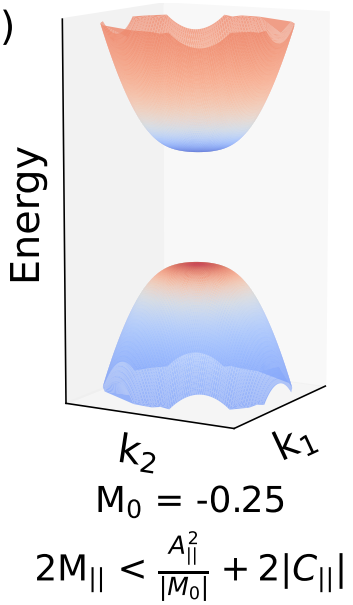

(d)

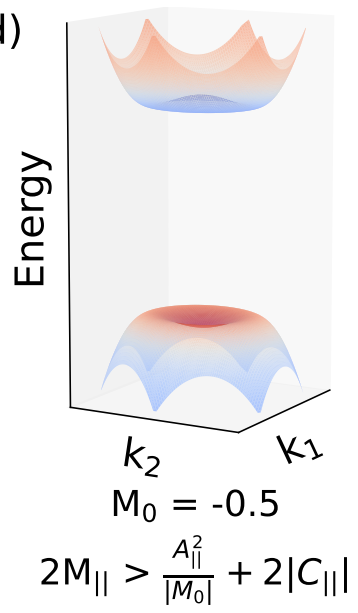

Fig. 2 Illustrations of the effects of varying the band inversion parameter $M_{0}$ on the valence and conduction bands, restricted to the L-plane (i.e. $k_{1}$ and $k_{2}$ span the L-plane). Blue and red represent the valence and conduction band characters prior to band inversion. The parameters of the $k \bullet p$ model are set as follows: $A_{\|}=1, M_{\|}=1.75$, and all other parameters are set to zero. Note that letting $C_{\|} \neq 0$ would make the conduction and valence bands asymmetric. (a) Normal, non-inverted parabolic bands $\left(M_{0}>0\right)$, reminiscent of conventional semiconductors. (b) When the gap closes, i.e. $M_{0}=0$, the bands are linear at the band crossing, forming a Dirac cone. (c) A gap is reopened when $M_{0}$ is slightly negative. Although inverted, the bands are still singly degenerate, as ensured by the condition $2 M_{\|}<\frac{A_{\|}^{2}}{\left|M_{0}\right|}+2\left|C_{\|}\right|$. (d) Ring-like band edges are induced when the $M_{0}$ parameter is decreased further such that the opposite condition $2 M_{\|}>\frac{A_{\|}^{2}}{\left|M_{0}\right|}+2\left|C_{\|}\right|$is satisfied.

verted (Figure 3b). The electronic structures of $\mathrm{PbTe}, \mathrm{PbSe}, \mathrm{PbS}$, and GeTe can therefore be represented by Figure 3a, whereas the electronic structure of the single-valleyed SnTe with inverted bands can be represented by Figure $3 \mathrm{~b}$. We obtain a ring-like Fermi surface (Figure 3c) when Equation (2) is satisfied. The ring-like conduction bands of GeSe and GeS (Figures 1c and S1e) can be modeled by Figure 3c. The ring-like Fermi surface can be split into six carrier pockets by including the $R_{1}$ parameter (Figure 3d), which can be further broken into three carrier pockets on each side of the L-plane by the $R_{2}$ parameter (Figure 3e). As a result, the $R_{1}$ and $R_{2}$ parameters enforce the three-fold rotational symmetry of the carrier pockets, similar to the hexagonal warping term used to model the surface states of $\mathrm{Bi}_{2} \mathrm{Te}_{3}$. ${ }^{33}$

It is interesting that rock-salt IV-VI compounds with inverted bands show no more than six carrier pockets, even though the $\overline{3} \mathrm{~m}$ symmetry of the L-point can permit up to twelve pockets. This can be understood by identifying the mirror planes of the L-point (Figure 4a); if the pockets are offset from the mirror planes, then there must be twelve pockets surrounding each L-point (Figure S3), as opposed to six when the pockets lie on the mirror planes (Figure 4b). We speculate that the chemical interactions which force the pockets to lie on the mirror planes are the same interactions as those which create the pockets at L in rock-salt IV-VI compounds. As described in Refs. 13 and 34, interactions between cation-p and anion-p states leads to both the valence and conduction band extrema at the L-point $\left(k_{x}=k_{y}=k_{z}= \pm \pi / a\right)$. These same interactions also affect the band energy when only two of $k_{x}$, $k_{y}$, or $k_{z}$ is $\pm \pi / a$, as evidenced by the second valence band maximum appearing along the $\Sigma$-line near $( \pm \pi / a, \pm \pi / a, 0){ }^{10,12}$ The $\mathrm{L}$ and $\Sigma$ pockets merge to form a tube-like Fermi surface approximately in the shape of a cube with corners $( \pm \pi / a, \pm \pi / a, \pm \pi / a),{ }^{35}$ as inscribed in the Brillouin zone in Figure $4 \mathrm{a}$. When band inver- sion forces the band extremum off L, the extremum then moves down the cube edge which lies on a mirror plane leading to six carrier pockets for each $L$ point.

\section{Chemical Origins of Band Inversion}

While many $k \bullet p$ parameters are involved in describing whether a rock-salt compound exhibits high valley degeneracy, the band inversion parameter $M_{0}$ in particular can be described in terms of chemical interactions directly through a tight-binding model. This is because the $M_{0}$ parameter is simply the difference between the conduction and valence band energies at the L-point, i.e.

$$
M_{0}=E_{C}(L)-E_{V}(L)
$$

for which analytical expressions can be derived from tight binding theory. ${ }^{13,34}$ By considering the s- and p-orbitals of the cation and anion as basis sets, the energies of the band edges at the Lpoint can be described in terms of on-site energies $(E)$ and orbital interactions $(V)$ as (using SnSe as the example IV-VI compound)

$$
\begin{aligned}
& E_{V}(\mathrm{~L})=\frac{1}{2}\left(E_{\mathrm{Se}_{\mathrm{p}}}^{\prime}+E_{\mathrm{Sn}_{\mathrm{s}}}\right)+\frac{1}{2} \sqrt{\left(E_{\mathrm{Se}_{\mathrm{p}}}^{\prime}-E_{\mathrm{Sn}_{\mathrm{s}}}\right)^{2}+48 V_{\mathrm{Sn}_{\mathrm{s}}-\mathrm{Se}_{\mathrm{p}}}^{2}} \\
& E_{C}(\mathrm{~L})=\frac{1}{2}\left(E_{\mathrm{Sn}_{\mathrm{p}}}^{\prime}+E_{\mathrm{Se}_{\mathrm{s}}}\right)+\frac{1}{2} \sqrt{\left(E_{\mathrm{Sn}_{\mathrm{p}}}^{\prime}-E_{\mathrm{Se}_{\mathrm{s}}}\right)^{2}+48 V_{\mathrm{Sn}_{\mathrm{p}}-\mathrm{Se}_{\mathrm{s}}}^{2}}
\end{aligned}
$$

for rock-salt SnSe, where

$$
\begin{aligned}
& E_{\mathrm{Se}_{\mathrm{p}}}^{\prime} \equiv E_{\mathrm{Se}_{\mathrm{p}}}+4\left(V_{\mathrm{Se}_{\mathrm{pp} \pi}}-V_{\mathrm{Se}} \mathrm{ep} \sigma\right) \\
& E_{\mathrm{Sn}_{\mathrm{p}}}^{\prime} \equiv E_{\mathrm{Sn}_{\mathrm{p}}}+4\left(V_{\mathrm{Sn}_{\mathrm{pp} \pi}}-V_{\mathrm{Sn}_{\mathrm{pp} \sigma}}\right)
\end{aligned}
$$

and analogous equations hold for the other rock-salt IV-VI compounds. ${ }^{13} E_{\mathrm{Se}_{\mathrm{p}}}$ is the on-site energy of the Se-p orbital, $V_{\mathrm{Sn}_{\mathrm{s}}}-\mathrm{Se}_{\mathrm{p}}$ is the nearest-neighbor Sn-s/Se-p interaction energy, and $V_{\mathrm{Se}_{\mathrm{pp} \sigma}}$ is 

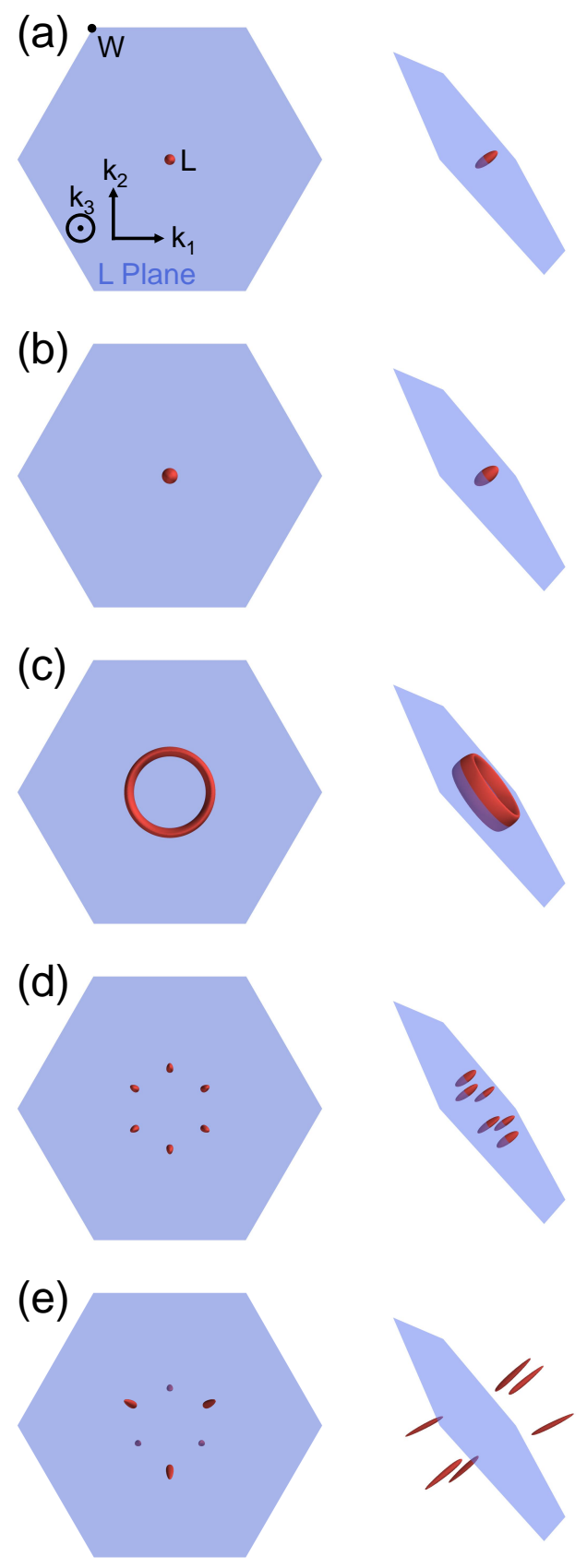

Fig. 3 Model Fermi surface geometries by varying $k \bullet p$ parameters in Equation (6). The basis vectors $k_{1}, k_{2}$, and $k_{3}$ of the $k \bullet p$ Hamiltonian are shown in (a). The blue hexagon corresponds to the L-plane of the Brillouin zone. The bands are not inverted in (a), whereas they are inverted in (b)-(e). The Fermi surfaces in (a), (b), and (c) are analogous to the band structures shown in Figures 2a, 2c, and $2 \mathrm{~d}$, respectively, with an additional out-of-plane component. In (d), the ring is warped into six Fermi surface pockets by the $R_{1}$ parameter. In (e), the pockets are lifted out of the L-plane by the $R_{2}$ parameter. Parameters used to plot these surfaces are listed in Table 1.

the next-nearest-neighbor Se-p/Se-p $\sigma$-interaction energy.

The molecular orbital energies at the L-point are drawn in Figure 5 , in accordance with the DFT results. Since the atomic orbital energies of the cation are higher than those of the anion, the conduction and valence band edges are primarily com-

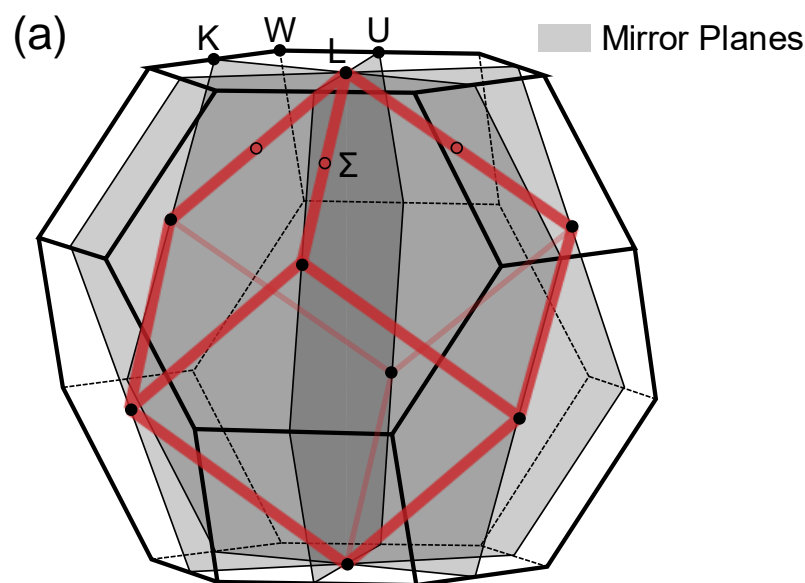

(b)

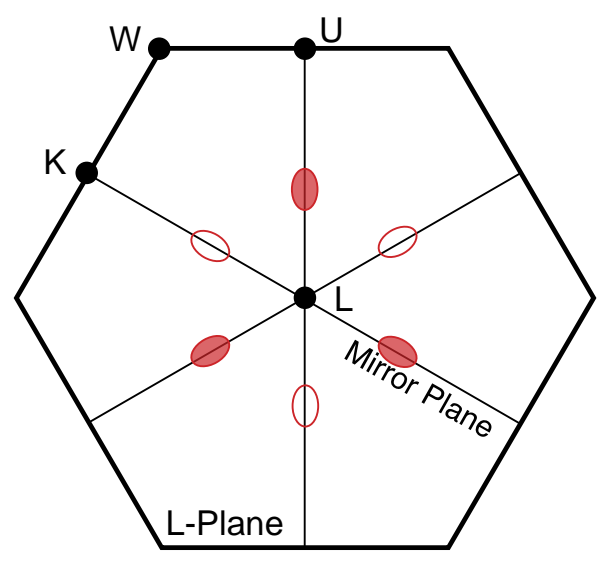

Fig. 4 (a) Brillouin zone of the rock-salt crystal structure, shown with mirror planes that pass through the L-point. High-symmetry $k$-points are shown as filled dots, and the approximate local maxima along the $\Sigma$ lines are shown as open dots. The cation- $p$ and anion-p orbital interactions in IV-VI compounds that lead to band extrema at the L-point ( $k_{x}=k_{y}=k_{z}= \pm \pi / a$ ) likely cause the band extrema to prefer the $\pi / a$ cube edges (two of $k_{x}, k_{y}$, or $k_{z}$ is $\pm \pi / a-$ drawn in red). ${ }^{13}$ (b) Six carrier pockets surrounding the L-point retaining $\overline{3} m$ symmetry when located on the mirror planes shown as solid lines. The filled and unfilled ellipses represent pockets located on opposite sides of the L-plane.

posed of cation-p and anion-p orbitals, respectively (Figure 5a). The molecular orbitals of the rock-salt IV-VI compounds arise from nearest-neighbor s/p-interactions between the cation and anion, as well as further splitting of the p-orbital energies by nextnearest-neighbor $\mathrm{p} / \mathrm{p}$-interactions. Cation- $\mathrm{p}$ and anion- $\mathrm{p}$ orbitals do not interact at the L-point due to symmetry restrictions. ${ }^{27}$ The band edge states have distinct symmetries, which we label $L_{6}^{-}$and $L_{6}^{+}$following the naming convention of Ref. 31 . The conduction and valence band edge states have $L_{6}^{-}$and $L_{6}^{+}$symmetry, respectively, when the bands are not inverted, whereas the symmetries are flipped when the bands are inverted (Figure 5b). ${ }^{27,30,31}$

It is clear from the diagram that one route to tune the band inversion parameter $M_{0}$ is by modulating the nearest-neighbor $\mathrm{s} / \mathrm{p}$ interaction. The conduction band edge state in a compound with normal band ordering results from the hybridization between cation-p and anion-s orbitals (Figure 5b), so decreasing the cation-p/anion-s interaction $V_{\mathrm{Sn}_{\mathrm{p}}-\mathrm{Se}_{\mathrm{s}}}$ would lower the energy of the $L_{6}^{-}$state. Similarly, the $L_{6}^{+}$valence band edge state results 
(a)

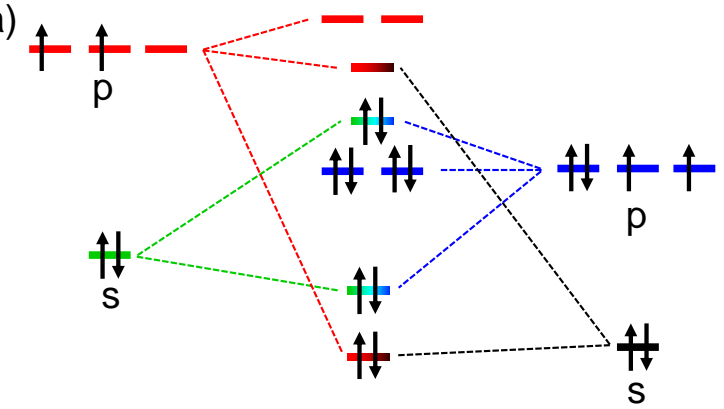

Cation IV-VI Compound Anion

(b)
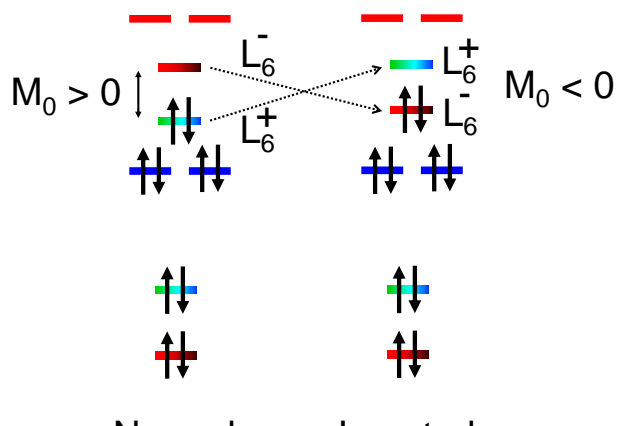

Normal Inverted

Fig. 5 (a) Hybridization between atomic orbitals of the cation and anion form the molecular orbitals of rock-salt IV-VI compounds. (b) Molecular orbital energies of non-inverted IV-VI compounds where the $L_{6}^{+}$state has lower energy than the $L_{6}^{-}$state, and inverted IV-VI compounds where the states are flipped. Nearest-neighbor s/p-interaction and next-nearest-neighbor $\mathrm{p} / \mathrm{p}$-splitting can be modified to tune the band inversion parameter $M_{0}$.

from the interaction between the anion-p and cation-s orbitals, so increasing the cation-s/anion-p interaction $V_{\mathrm{Sn}_{\mathrm{s}}}-\mathrm{Se}_{\mathrm{p}}$ would raise the energy of the $L_{6}^{+}$state. This can also be seen from Equation (4). Tuning the interaction energies in such a way will result in the inversion of the $L_{6}^{-}$and $L_{6}^{+}$states (Figure $5 \mathrm{~b}$ ).

Tuning the chemical interaction strengths to achieve high valley degeneracy can be done in several ways, for example by alloying and mechanical deformation. To induce multiple carrier pockets in SnTe, we suggest alloying the compound with either SnSe or SnS. While SnSe and SnS natively crystallize in the layered orthorhombic Pnma structure, ${ }^{36,37}$ single-phase alloys between SnTe and SnSe in the rock-salt structure have been reported, ${ }^{38,39}$ indicating that the rock-salt crystal structure can be maintained up to a solubility limit. Given that the s- and p-orbital energies of Se and S are lower than those of Te, ${ }^{40}$ we would expect the Sns/anion-p interaction to strengthen since the atomic orbital energies will be closer, resulting in an increase in the energy of the $L_{6}^{+}$state. Similarly, we would expect the Sn-p/anion-s interaction to weaken since the energy separation between the Sn-p and the anion-s atomic orbitals will be larger, resulting in a decrease in the energy of the $L_{6}^{-}$state. While it is certainly not guaranteed that simply increasing/decreasing the chemical interactions will lead to multiple carrier pockets, we emphasize that exaggerating the interaction strengths to invert the bands enough is the key criteria for engineering high valley degeneracy. We suggest revisiting such alloys in light of the present study, as our model predicts a $6 \times$ increase in the valley degeneracy of SnTe to $N_{V}=24$.

\section{Conclusion}

Our analysis of the electronic structures of rock-salt IV-VI compounds shows that the mere existence of inverted bands is an insufficient condition for high valley degeneracy near the band edges. Instead, the $k \bullet p$ model of the L-point in rock-salt IV-VI compounds reveals that the degree to which the bands are inverted is a far more relevant property to consider. Our model predicts that a $6 \times$ increase in the valley degeneracy, from $N_{V}=4$ to $N_{v}=24$, can be achieved in rock-salt IV-VI compounds with inverted bands by tuning nearest-neighbor chemical interactions.

It is worth mentioning however that while the $z T$ is expected to increase with valley degeneracy, intervalley scattering mechanisms (which reduce the benefit of $N_{V}$ ) may become nonnegligible when multiple carrier pockets are present. ${ }^{7,41,42}$ As this is outside the scope of the present study, we leave the effects of intervalley scattering in compounds with multiple carrier pockets to a future study. Our analysis also holds for materials in which band inversion occurs at a $k$-point with $\overline{3} m$ point group symmetry. Notably, our model explains the dissimilar Fermi surface geometries of $\mathrm{Bi}_{2} \mathrm{Te}_{3}$ and $\mathrm{Bi}_{2} \mathrm{Se}_{3}$, where the $k$-point at which band inversion occurs $(\Gamma)$ also has $\overline{3} m$ point group symmetry. Our analysis suggests that one reason why $\mathrm{Bi}_{2} \mathrm{Te}_{3}$ exhibits high valley degeneracy whereas $\mathrm{Bi}_{2} \mathrm{Se}_{3}$ exhibits single valley degeneracy is because the bands are sufficiently inverted in $\mathrm{Bi}_{2} \mathrm{Te}_{3}$. Even though band inversion exists in $\mathrm{Bi}_{2} \mathrm{Se}_{3}$, it is not enough to move the band extrema off the $\Gamma$ point. ${ }^{18,19}$ In general, although the specific Fermi surface geometry depends on complex interactions, there appears to exist a critical degree of inversion, below which the Fermi surface remains single-valleyed.

\section{Methods}

\section{Density Functional Theory Calculations}

Density Functional Theory calculations were performed using the Vienna ab-initio Simulation Package (VASP) ${ }^{43-45}$ using the projector augmented wave (PAW) method. ${ }^{46,47}$ The Perdew-BurkeErnzerhof (PBE) functional ${ }^{48}$ was used in each calculation with a plane-wave energy cutoff of $500 \mathrm{eV}$ and k-point grid of $33 \times$ $33 \times 33$ (6001 irreducible k-points) ${ }^{49}$ for all electronic structure calculations. Spin-orbit coupling was included in all calculations. The Fermi surfaces were calculated by interpolating the electronic structure on a k-point grid that is 5 times as dense using the iFermi software. ${ }^{50}$

\section{$k \bullet p$ Model}

The electronic structure of rock-salt IV-VI compounds at the Lpoint can be well-described using the method of invariants of $k \bullet p$ perturbation theory. ${ }^{28,51,52}$ The L-point has $\overline{3} m$ point group symmetry. ${ }^{53}$ We choose the normalized reciprocal lattice basis vectors to be $k_{1} \equiv\left(-\frac{1}{\sqrt{2}}, \frac{1}{\sqrt{2}}, 0\right), k_{2} \equiv\left(-\frac{1}{\sqrt{6}},-\frac{1}{\sqrt{6}}, \frac{2}{\sqrt{6}}\right)$, and $k_{3} \equiv$ $\left(\frac{1}{\sqrt{3}}, \frac{1}{\sqrt{3}}, \frac{1}{\sqrt{3}}\right)$ centered at the L-point. By using the valence and conduction band edges as basis functions and including SOC (i.e. $|\mathrm{VBM}, \uparrow\rangle, \mathrm{CBM}, \uparrow\rangle, \mathrm{VBM}, \downarrow\rangle$, and CBM, $\downarrow\rangle)$, the effective four-band 
$k \bullet p$ Hamiltonian up to third order in $k$ is 54,55

$$
\begin{aligned}
H_{\text {eff }} & =\left(\begin{array}{cccc}
\varepsilon_{\mathbf{k}}+M(\mathbf{k}) & \mathscr{A}_{\perp}\left(k_{3}\right) k_{3} & 0 & \mathscr{A}_{||}\left(k_{\|}\right) k_{-} \\
\mathscr{A}_{\perp}\left(k_{3}\right) k_{3} & \varepsilon_{\mathbf{k}}-M(\mathbf{k}) & \mathscr{A}_{\mid}\left(k_{\|}\right) k_{-} & 0 \\
0 & \mathscr{A}_{||}\left(k_{\|}\right) k_{+} & \varepsilon_{\mathbf{k}}+M(\mathbf{k}) & -\mathscr{A}_{\perp}\left(k_{3}\right) k_{3} \\
\mathscr{A}_{||}\left(k_{\|}\right) k_{+} & 0 & -\mathscr{A}_{\perp}\left(k_{3}\right) k_{3} & \varepsilon_{\mathbf{k}}-M(\mathbf{k})
\end{array}\right) \\
& +\frac{R_{1}\left(k_{+}^{3}+k_{-}^{3}\right)}{2}\left(\begin{array}{cccc}
0 & i & 0 & 0 \\
-i & 0 & 0 & 0 \\
0 & 0 & 0 & i \\
0 & 0 & -i & 0
\end{array}\right) \\
& +\frac{R_{2}\left(k_{+}^{3}-k_{-}^{3}\right)}{2}\left(\begin{array}{cccc}
0 & -i & 0 & 0 \\
-i & 0 & 0 & 0 \\
0 & 0 & 0 & i \\
0 & 0 & i & 0
\end{array}\right)
\end{aligned}
$$

where

$$
\begin{aligned}
\varepsilon_{\mathbf{k}} & \equiv C_{0}+C_{\perp} k_{3}^{2}+C_{\|} k_{\|}^{2} \\
M(\mathbf{k}) & \equiv M_{0}+M_{\perp} k_{3}^{2}+M_{\|} k_{\|}^{2} \\
\mathscr{A}_{\|}\left(k_{\|}\right) & \equiv A_{\|}+A_{\|}^{\prime} k_{\|}^{2} \\
\mathscr{A}_{\perp}\left(k_{3}\right) & \equiv A_{\perp}+A_{\perp}^{\prime} k_{3}^{2} \\
k_{\|}^{2} & \equiv k_{1}^{2}+k_{2}^{2} \\
k_{ \pm} & \equiv k_{1} \pm i k_{2}
\end{aligned}
$$

The last two terms in Equation (6) are analogous to the hexagonal warping terms introduced in Ref. 33, as they break the ring-like Fermi surface in Figure $3 c$ to six carrier pockets as in Figures 3d and $3 \mathrm{e}$. Note that the band edges are doubly degenerate due to inversion symmetry and time-reversal symmetry, ${ }^{56}$ which permits an analytical model for the energy bands

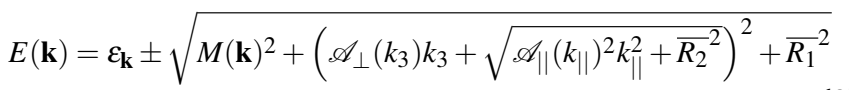

where

$$
\begin{aligned}
& \overline{R_{1}} \equiv R_{1}\left(k_{1}^{3}-3 k_{1} k_{2}^{2}\right) \\
& \overline{R_{2}} \equiv R_{2}\left(3 k_{1}^{2} k_{2}-k_{2}^{3}\right)
\end{aligned}
$$

\section{High Valley Degeneracy Condition on the L-plane}

We consider paths on the L-plane by setting $k_{3}=0$. Starting with the conduction band, Equation (8) simplifies to

$$
E_{C}(\mathbf{k})=\varepsilon_{\mathbf{k}}+\sqrt{M(\mathbf{k})^{2}+\mathscr{A}_{\|}\left(k_{\|}\right)^{2} k_{\|}^{2}+{\overline{R_{1}}}^{2}+{\overline{R_{2}}}^{2}}
$$

To obtain a ring-like conduction band minimum around the Lpoint, the conduction band must attain a local maximum at the Lpoint. This condition is guaranteed by a negative-definite Hessian matrix $\hat{H}$ of $E_{C}(\mathbf{k})$ at the L-point, where

$$
\hat{H}=\left(\begin{array}{cc}
\frac{\partial^{2} E_{C}}{\partial k_{1}^{2}} & \frac{\partial^{2} E_{C}}{\partial k_{1} \partial k_{2}} \\
\frac{\partial^{2} E_{C}}{\partial k_{2} \partial k_{1}} & \frac{\partial^{2} E_{C}}{\partial k_{2}^{2}}
\end{array}\right) .
$$

$\hat{H}$ is negative-definite if the eigenvalues of the matrix are negative. It turns out that the diagonal terms are identical and are the only nonzero elements in $\hat{H}$, where

$$
\left.\frac{\partial^{2} E_{C}}{\partial k_{1}^{2}}\right|_{\left(k_{1}, k_{2}\right)=(0,0)}=\left.\frac{\partial^{2} E_{C}}{\partial k_{2}^{2}}\right|_{\left(k_{1}, k_{2}\right)=(0,0)}=2 C_{\|}-\left(2 M_{\|}-\frac{A_{\|}^{2}}{\left|M_{0}\right|}\right)
$$

such that the condition shown in Equations (1) and (2) can be derived. A similar logic follows for the valence band, where the eigenvalues of the Hessian matrix of $E_{V}(\mathbf{k})$ at the L-point must be positive for a ring-like valence band maximum.

Table $1 k \bullet p$ parameters used to plot Figure 3. All unlisted parameters are set to zero for all subplots.

\begin{tabular}{cccccc} 
Parameter & (a) & (b) & (c) & (d) & (e) \\
\hline $\mathrm{M}_{0}$ & 0.1 & -0.1 & -0.1 & -0.1 & -0.1 \\
$\mathrm{M}_{\|}$ & 40 & 0 & 40 & 40 & 40 \\
$\mathrm{M}_{\perp}$ & 5 & 0 & 0 & 0 & 0 \\
$\mathrm{~A}_{\|}$ & 0 & 2 & 2 & 2 & 2 \\
$\mathrm{~A}_{\perp}$ & 0 & 1 & 1 & 1 & 1 \\
$\mathrm{R}_{1}$ & 0 & 0 & 0 & 1000 & 1000 \\
$\mathrm{R}_{2}$ & 0 & 0 & 0 & 0 & -1000 \\
\hline
\end{tabular}

\section{Acknowledgements}

M.Y.T. is funded by the United States Department of Energy through the Computational Science Graduate Fellowship (DOE CSGF) under grant number DE-SC0020347. M.K.B., L.G., E.E. and G.J.S. acknowledges support from NSF DMREF grant number 1729487. F.A.B. acknowledges support from NSF DIGI-MAT program, grant number 1922758. B.A.A. acknowledges support from NSF-DMR-1905277. This research was supported in part through the computational resources and staff contributions provided for the Quest high performance computing facility at Northwestern University, which is jointly supported by the Office of the Provost, the Office for Research, and Northwestern University Information Technology.

\section{References}

1 J. Xin, Y. Tang, Y. Liu, X. Zhao, H. Pan and T. Zhu, npj Quant. Mater., 2018, 3, 1.

2 Y. Pei, H. Wang and G. J. Snyder, Adv. Mater., 2012, 24, 6125.

3 W. Liu, X. Tan, K. Yin, H. Liu, X. Tang, J. Shi, Q. Zhang and C. Uher, Phys. Rev. Lett., 2012, 108, 166601.

4 Y. Tang, Z. M. Gibbs, L. A. Agapito, G. Li, H.-S. Kim, M. B. Nardelli, S. Curtarolo and G. J. Snyder, Nat. Mater., 2015, 14, 1223.

5 R. Hanus, X. Guo, Y. Tang, G. Li, G. J. Snyder and W. G. Zeier, Chem. Mater., 2017, 29, 1156.

6 P. R. Raghuvanshi, S. Mondal and A. Bhattacharya, J. Mater. Chem. A, 2020, 8, 25187. 
7 H. Wang, Y. Pei, A. D. LaLonde and G. J. Snyder, Thermoelectric Nanomaterials, Springer, 2013, p. 3.

8 A. D. LaLonde, Y. Pei, H. Wang and G. J. Snyder, Mater. Today, 2011, 14, 526.

9 Y. Pei, X. Shi, A. LaLonde, H. Wang, L. Chen and G. J. Snyder, Nature, 2011, 473, 66.

10 L.-D. Zhao, H. J. Wu, S. Q. Hao, C.-I. Wu, X. Y. Zhou, K. Biswas, J. Q. He, T. P. Hogan, C. Uher, C. Wolverton, V. P. Dravid and M. G. Kanatzidis, Energ. Environ. Sci., 2013, 6, 3346.

11 P. Jood, J. P. Male, S. Anand, Y. Matsushita, Y. Takagiwa, M. G. Kanatzidis, G. J. Snyder and M. Ohta, J. Am. Chem. Soc., 2020, 142, 15464.

12 S. Ahmad, A. Singh, S. Bhattacharya, M. Navaneethan, R. Basu, R. Bhatt, P. Sarkar, K. N. Meshram, A. K. Debnath, K. P. Muthe and D. K. Aswal, ACS Appl. Energ. Mater., 2020, 3, 8882 .

13 M. K. Brod and G. J. Snyder, J. Mater. Chem. A, 2021, 9, 12119.

14 H.-X. Wang, L.-S. Mao, X. Tan, G.-Q. Liu, J. Xu, H. Shao, H. Hu and J. Jiang, Nano Energy, 2018, 51, 649.

15 D. Wang, W. He, C. Chang, G. Wang, J. Wang and L.-D. Zhao, J. Mater. Chem. C, 2018, 6, 12016.

16 G. Xie, Z. Li, T. Luo, H. Bai, J. Sun, Y. Xiao, L.-D. Zhao, J. Wu, G. Tan and X. Tang, Nano Energy, 2020, 69, 104395.

17 H. Shi, D. Parker, M.-H. Du and D. J. Singh, Phys. Rev. Appl., 2015, 3, 014004.

18 J. P. Heremans, R. J. Cava and N. Samarth, Nat. Rev. Mater., 2017, 2, 1 .

19 I. T. Witting, F. Ricci, T. C. Chasapis, G. Hautier and G. J. Snyder, Research, 2020, 2020, 1.

20 I. T. Witting, T. C. Chasapis, F. Ricci, M. Peters, N. A. Heinz, G. Hautier and G. J. Snyder, Adv. Electron. Mater., 2019, 5, 1800904.

21 M. Z. Hasan and C. L. Kane, Rev. Mod. Phys., 2010, 82, 3045.

22 Y. L. Chen, J. G. Analytis, J.-H. Chu, Z. K. Liu, S.-K. Mo, X. L. Qi, H. J. Zhang, D. H. Lu, X. Dai, Z. Fang, S. C. Zhang, I. R. Fisher, Z. Hussain and Z.-X. Shen, Science, 2009, 325, 178.

23 Y. Tanaka, Z. Ren, T. Sato, K. Nakayama, S. Souma, T. Takahashi, K. Segawa and Y. Ando, Nat. Phys., 2012, 8, 800.

24 Y. Zhang, Z. Liu, B. Zhou, Y. Kim, L. Yang, H. Ryu, C. Hwang, Y. Chen, Z. Hussain, Z.-X. Shen and S.-K. Mo, J. Electron Spectrosc., 2017, 219, 35.

25 I. Tateishi and H. Matsuura, J. Phys. Soc. Jpn., 2018, 87, 073702.

26 H. Zhu, W. Sun, R. Armiento, P. Lazic and G. Ceder, Appl. Phys. Lett., 2014, 104, 082107.

27 Z.-Y. Ye, H.-X. Deng, H.-Z. Wu, S.-S. Li, S.-H. Wei and J.-W. Luo, npj Comput. Mater., 2015, 1, 1.

28 L. C. L. Y. Voon and M. Willatzen, The $k \bullet p$ method: electronic properties of semiconductors, Springer Science \& Business Me- dia, 2009.

29 J. O. Dimmock and G. B. Wright, Phys. Rev., 1964, 135, A821.

30 J. Conklin Jr, L. Johnson and G. Pratt Jr, Phys. Rev., 1965, 137, A1282.

31 J. Dimmock, I. Melngailis and A. Strauss, Phys. Rev. Lett., 1966, 16, 1193.

32 D. L. Mitchell and R. F. Wallis, Phys. Rev., 1966, 151, 581.

33 L. Fu, Phys. Rev. Lett., 2009, 103, 266801.

34 M. K. Brod, M. Y. Toriyama and G. J. Snyder, Chem. Mater, 2020, 32, 9771.

35 P. B. Littlewood, B. Mihaila, R. K. Schulze, D. J. Safarik, J. E. Gubernatis, A. Bostwick, E. Rotenberg, C. P. Opeil, T. Durakiewicz, J. L. Smith and J. C. Lashley, Phys. Rev. Lett., 2010, 105, 086404.

36 L.-D. Zhao, S.-H. Lo, Y. Zhang, H. Sun, G. Tan, C. Uher, C. Wolverton, V. P. Dravid and M. G. Kanatzidis, Nature, 2014, 508, 373.

37 Asfandiyar, B. Cai, L.-D. Zhao and J.-F. Li, J. Mater., 2020, 6, 77.

38 B. R. Ortiz, J. M. Adamczyk, K. Gordiz, T. Braden and E. S. Toberer, Mol. Syst. Des. Eng., 2019, 4, 407.

39 X. Guo, Z. Chen, J. Tang, F. Zhang, Y. Zhong, H. Liu and R. Ang, Appl. Phys. Lett., 2020, 116, 103901.

40 W. A. Harrison, Electronic structure and the properties of solids: the physics of the chemical bond, Courier Corporation, 2012.

41 P. Norouzzadeh and D. Vashaee, Sci. Rep., 2016, 6, 1.

42 J. Park, M. Dylla, Y. Xia, M. Wood, G. J. Snyder and A. Jain, Nat. Commun., 2021, 12, 1.

43 G. Kresse, J. Non-Cryst. Solids, 1995, 192, 222.

44 G. Kresse and J. Furthmüller, Comp. Mater. Sci., 1996, 6, 15.

45 G. Kresse and J. Furthmüller, Phys. Rev. B, 1996, 54, 11169.

46 P. E. Blöchl, Phys. Rev. B, 1994, 50, 17953.

47 G. Kresse and D. Joubert, Phys. Rev. B, 1999, 59, 1758.

48 J. P. Perdew, K. Burke and M. Ernzerhof, Phys. Rev. Lett., 1996, 77, 3865.

49 H. J. Monkhorst and J. D. Pack, Phys. Rev. B, 1976, 13, 5188.

50 A. M. Ganose, A. Searle, A. Jain and S. M. Griffin, Journal of Open Source Software, 2021, 6, 3089.

51 J. Luttinger, Phys. Rev., 1956, 102, 1030.

52 P. Y. Yu and M. Cardona, Fundamentals of semiconductors: physics and materials properties, Springer Science \& Business Media, 2010.

53 T. H. Hsieh, H. Lin, J. Liu, W. Duan, A. Bansil and L. Fu, Nat. Commun., 2012, 3, 1.

54 H. Zhang, C.-X. Liu, X.-L. Qi, X. Dai, Z. Fang and S.-C. Zhang, Nat. Phys., 2009, 5, 438.

55 C.-X. Liu, X.-L. Qi, H. Zhang, X. Dai, Z. Fang and S.-C. Zhang, Phys. Rev. B, 2010, 82, 045122.

56 L. Petersen and P. Hedegård, Surf. Sci., 2000, 459, 49. 